\title{
Anticancer nanometre-biomissiles carrying a leucine insulator and an antibody complementarity-determining region
}

\author{
Meng Xing ${ }^{1}$, Xiaoxia $\mathrm{Li}^{2}$, Shanshan An ${ }^{1}$, Chongfeng Lan ${ }^{1}$, Min Fu ${ }^{1}$, Yunfan Shi ${ }^{1}$, \\ Yan Huang ${ }^{3}$, Man Tang ${ }^{1}$, Yulin Wan ${ }^{1}$, Yuchuan Wang ${ }^{4}$, Jingli Peng ${ }^{1}$, \\ Kai Wang ${ }^{1}$, Zi-Wei Ye ${ }^{5}$, Shaoping Weng ${ }^{1}$, Qiuyun Liu ${ }^{1} *$, Jianguo $\mathrm{He}^{1}$ and \\ Wenliang Zhou ${ }^{1}$
}

${ }^{1}$ State Key Laboratory of Biocontrol, Key Laboratory for Improved Variety Reproduction of Aquatic Economic Animals of Guangdong Province, Biomedical Center, School of Life Sciences, Sun Yat-sen University, Guangzhou 510275, China

${ }^{2}$ School of Materials Science and Engineering, Sun Yat-Sen University, Guangzhou 510275, China

${ }^{3}$ Division of Life Science, Hong Kong University of Science and Technology, Clear water bay, Kowloon, Hong Kong

${ }^{4}$ Center for Synthetic Biology Engineering Research, Shenzhen Institutes of Advanced Technology, Chinese Academy of Sciences,

Shenzhen, Guangdong 518055, China

${ }^{5}$ Department of Microbiology, The University of Hong Kong, Hong Kong

\begin{abstract}
The tumour specificities and potency of miniaturized cancer targeting peptides are among the major issues of current cancer research. Over 100 peptides were evaluated in this study. According to the observations, leucine could sustain the antibacterial and anticancer efficacy of (leucine/lysine)n ((L/K)n) peptides across different assay conditions, whereas (valine/lysine) $n$ $((\mathrm{V} / \mathrm{K}) n)$ and (isoleucine/lysine $) n((\mathrm{I} / \mathrm{K}) n)$ peptides were less effective. Tumour targeting peptides, consisting of a leucine/lysine peptide and an antibody complementarity-determining region (CDR) fragment against CD47 receptor separated by a leucine 4 insulator, eradicated $100 \%$ of the lung cancer A549 cells at $100 \mu \mathrm{m}$ concentration after $24 \mathrm{~h}$ of incubation. It also inhibited tumour growth in a mouse model of colon cancer cells, showing extensive apoptosis at the end of the treatment. Our data suggest that leucine insulators could be effective spacers in the design of targeted or plurispecific peptide drugs given the lack of $\sigma-\sigma$ hyperconjugation on $\beta$-carbon and the lack of strong van der Waals interactions between the side group and the carbonyl group. Significant reduction of hemolysis by $\mathbf{P 4 5 - 3}$ with terminal alanine replacement on peptide P129 suggests that biosafety attribute of a peptide can be substantially improved.
\end{abstract}

Keywords: Anticancer nanometre-biomissiles, antibody complementarity-determining region, consensus peptide sequences, leucine insulator, mouse model.

ANTIMICROBIAL peptides (AMPs) are ubiquitous in the animal and plant kingdoms, and are evolutionarily conserved components of innate immune response ${ }^{1,2}$. AMPs

*For correspondence. (e-mail: 1sslqy@ mail.sysu.edu.cn) can be divided into four major classes based on secondary structures: $\alpha$-helical, $\beta$-sheet, loop and extended peptides. The activities of several AMPs are sensitive to salt concentrations $^{2}$, which reduces their practical applications. The design of artificial AMPs can be an advantage as they may avoid the stresses AMPs generate in the host cells, consequently circumventing the problem of attenuation of AMPs through evolution. Based on the chemical perspective for leucine and $\beta$-branched valine and isoleucine (Figure 1) $)^{3-8}$, a large number of peptides were designed to determine the biological activities for antibacterial and anticancer purposes. Numerous organic acids generated from the Krebs cycle or other pathways possess modest median lethal doses on animals and are negatively charged at physiological $\mathrm{pH}$. Could they also be involved in the cell disruption mechanism for peptides? This study was prompted by the need to understand the mechanism of salt-sensitivity of AMPs and to design active artificial AMPs in the treatment of cancer, bacterial infections, etc. Observations were made on synthetic peptides and their effects on model cell lines and pathological microbes. We found that $\beta$-branched valine with 2 $\gamma$-methyl groups and to a less extent isoleucine with a single $\gamma$-methyl group were among the key factors in the attenuation of AMPs, and non- $\beta$-branched leucines could sustain the anticancer and antibacterial activities of (leucine/lysine $) n((\mathrm{~L} / \mathrm{K}) n)$ peptides.

\section{Materials and methods}

\section{Bacterial strains and reagents}

Pseudomonas aerugenosa (1.2464) was purchased from China General Microbiological Culture Collection Center 
(CGMCCC), Beijing, China. The multidrug-resistant Staphylococcus aureus Y5 strain has been described in a previous study ${ }^{9}$. Escherichia coli MG1655 was a kind gift from the E. coli Genetic Stock Center (Yale University, New Heaven, CT, USA). S. aureus (ATCC6538) was obtained from Guangdong Microbiology Culture Center, Guangzhou, China. Reagents were of analytical grade.

\section{Culture media and equipment}

Ten per cent fetal bovine serum (FBS) was obtained from Hyclone (GE Healthcare Life Sciences Inc, Utah, USA), and $1 \%$ antibiotics pen-strep was a product of Gibco (Thermo Fisher Scientific Inc, MA, USA). The tumour tissues were observed using a microscope (Olympus BX51WI, Japan). Fluorescence of FITC-labelled peptide in the treatment of bacterial cells was visualized with a confocal laser scanning microscope (Zeiss7 DUO NLO, Zeiss, Jena, Germany). Bacterial cells were examined employing transmission electron microscopy (TEM; JEM 1400 electron microscope, JEOL Ltd, Japan). Scanning electron microscopy (SEM) of cancer cells was also performed (Hitachi S-3400N, Japan).

\section{Peptide synthesis}

Solid-phase peptide synthesis was performed by Shanghai BOOTECH Bioscience and Technology Co, Ltd. The peptides were synthesized employing the Fmoc strategy and purified using reversed-phase-high-performance liquid chromatography (RP-HPLC). Characterizations were performed using HPLC and mass spectral analysis. Peptides were at least $98 \%$ or $95 \%$ pure. Cecropin A was amidated at the carboxyl terminus ${ }^{10}$. Magainin 2 was synthesized as an additional control ${ }^{11}$.

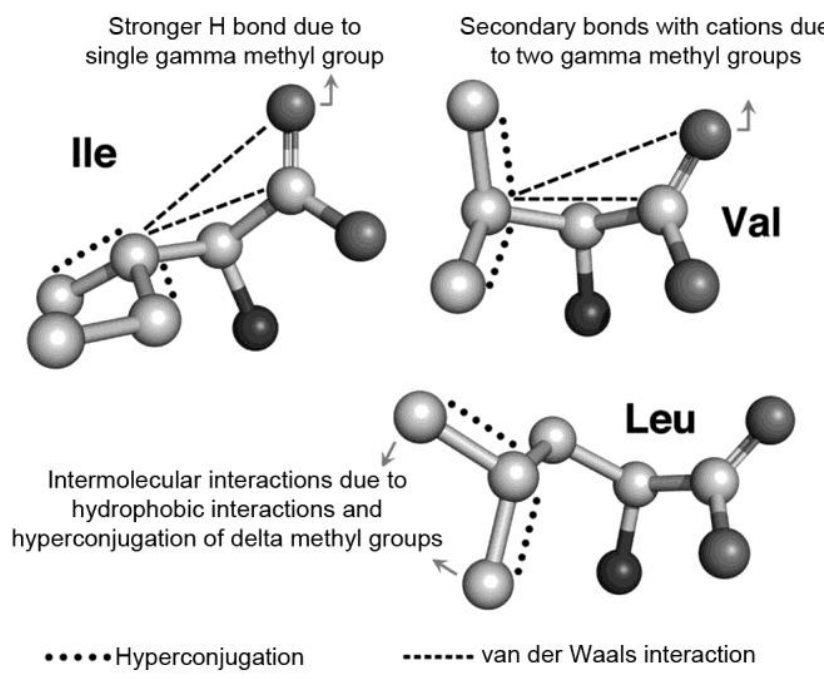

Figure 1. Model depicting chemical perspectives of leucine, isoleucine and valine supported by NMR chemical shift anisotropy and other data.

\section{Cell culture and cell viability assay}

Human bronchial epithelial 16HBE14o-cells ${ }^{12}$ and lung carcinoma A549 cells ${ }^{13}$ were cultured in DMEM/F12 and DMEM (high glucose) respectively, as recommended by the American Type Culture Collection. Both media were supplemented with $10 \%$ FBS. Cell viability was determined using the 3-(4,5-dimethylthiazol-2yl)-2,5-diphenyl-tetrazolium bromide (MTT; MBCHAM) colorimetric dye method, as previously described ${ }^{14}$. In brief, 16HBE14oand A549 cells were plated overnight in 96-well plates. Then the cells were treated with various peptides and concentrations up to $24 \mathrm{~h}$. The inhibition rate of cell viability was measured using the formula

Cell viability inhibition rate $(\%)=$

$\frac{\text { Control value A490 - Experimental value A490 }}{\text { Control value A490 }} \times 100 \%$.

All experimental protocols related to human cells and human tissue in this study were approved by the Committee of the School of Life Sciences at Sun Yat-Sen University, China, and all methods were carried out in accordance with the approved guidelines. Informed consent was obtained from the blood donors (including one of the present author M.T.) for haemolytic assay in accordance with the Declaration of Helsinki. Human lung carcinoma A549 cells were provided by Jun Xu (State Key Laboratory of Respiratory Disease, Guangzhou, China). Human bronchial epithelial 16HBE14o-cells were generously provided by Steven Holgate (University of Southampton, England). The mouse study was performed in strict accordance with the recommendations in the Guide for the Care and Use of Laboratory Animals of the National Institutes of Health, USA. The protocol was approved by the Committee on the Ethics of Animal Experiments of Sun Yat-Sen University. Mice were sacrificed by cervical vertebra luxation.

\section{HT29 subcutaneous transplantation tumour animal models}

All animal experiments were approved by the Institutional Animal Care and Use Committee of the Sun Yat-sen University. Female Nu/Nu nude mice (Beijing Vital River Laboratories), which were 6-7 weeks and randomized into batches of four in control and five in peptide treatment group, were subcutaneously injected with $1 \times 10^{7}$ HT29 cells on the right abdomen. When the tumour volume had grown up to about $100 \mathrm{~mm}^{3}, 200 \mu \mathrm{l} 6 \mathrm{mg} / \mathrm{ml}$ peptide P129 was intravenously injected via the tail vein of the treatment mice every other day from day 1 to day 21 , while those in the control group underwent the same procedure with phosphate buffer solution (PBS) simultaneously. The weight of the mice and tumour volume were measured with a vernier calliper every two days from 
day 1 to day 21, and the tumour volumes were calculated by the following formula

$$
V=\pi / 6 \times a \times b^{2},
$$

where $a$ is the length and $b$ is the width of tumor.

After treatment with the peptide, the mice were sacrificed and tumour tissues were subjected to haematoxylin/eosin (H\&E) staining assay and TUNEL assay. The $\mathrm{H} \& \mathrm{E}$ staining operations were as follows: the paraffin sections were placed at $60^{\circ} \mathrm{C}$ atmosphere for $2 \mathrm{~h}$, dewaxed twice in xylene and subsequently were immersed in $100 \%, 90 \%, 80 \%$ and $70 \%$ ethanol respectively. Staining experiments were in strict accordance with the manuals of KeyGEN H\&E staining kits. After dehydration in $100 \%$ ethanol, the specimens were placed in xylene to obtain transparency; the tumour tissues were observed under a microscope and photographed after being covered with neutral gum.

The TUNEL assay was performed using Merck FragEL $^{\mathrm{TM}}$ DNA Fragmentation Detection $\mathrm{Kit}^{15}$. The procedure was as follows: the dewaxing and hydration operation steps of the paraffin sections were the same as $\mathrm{H} \& \mathrm{E}$ staining. Then $2 \mathrm{mg} / \mathrm{ml}$ Proteinase $\mathrm{K}$ was diluted to $20 \mu \mathrm{g} / \mathrm{ml}$ in $10 \mathrm{nM}$ Tris $\mathrm{pH}$ 8.0. Slides were rinsed with $1 \times$ TBS and the tissue sections were covered with $100 \mu \mathrm{l}$ of $20 \mu \mathrm{g} / \mathrm{ml}$ proteinase K. Specimens were incubated at room temperature for $20 \mathrm{~min}$. Slides were subsequently rinsed with $1 \times$ TBS. Next $30 \% \mathrm{H}_{2} \mathrm{O}_{2}$ was diluted ten-fold in methanol and the tissue sections were covered with $100 \mu \mathrm{l}$ of $3 \% \mathrm{H}_{2} \mathrm{O}_{2}$ followed by incubation at room temperature for $5 \mathrm{~min}$. After rinsing the slides with $1 \times \mathrm{TBS}$, the tissue sections were covered with $100 \mu \mathrm{l}$ of $1 \times \mathrm{TdT}$ equilibration buffer followed by incubation at room temperature for $20 \mathrm{~min}$. Next $57 \mu \mathrm{l}$ TdT labelling reaction mix was mixed gently with $3 \mu \mathrm{TdT}$ enzyme on ice for each sample. The $1 \times$ TdT equilibration buffer was carefully blotted from the tissue section and $60 \mu \mathrm{l}$ of TdT labelling reaction mixture was immediately applied onto each sample and the specimens were covered with a piece of parafilm. Slides were placed in a humidified chamber and incubated at $37^{\circ} \mathrm{C}$ for $1.5 \mathrm{~h}$. They were rinsed with $1 \times \mathrm{TBS}$ and the tissue sections were covered with $100 \mu \mathrm{l}$ of blocking buffer, followed by incubation at room temperature for $10 \mathrm{~min}$. The blocking buffer was carefully blotted from the tissue sections and $100 \mu \mathrm{l}$ of diluted $1 \times$ conjugate was immediately applied to the tissue section. Slides were placed in a humidified chamber and incubated at room temperature for $30 \mathrm{~min}$. They were rinsed with $1 \times$ TBS and the entire tissue section was covered with $100 \mu \mathrm{l}$ of $3,3^{\prime}$-diaminobenzidine (DAB) solution for $10 \mathrm{~min}$. Slides were rinsed with running water. The tissue section was covered with $100 \mu$ l of methyl green counterstain solution for $30 \mathrm{sec}$ and the slides were immediately rinsed with running water. The glass slides were dried and mounted on a glass coverslip using neutral gum.

\section{Confocal laser scanning microscopy of bacterial cells}

E. coli MG1655 and S. aureus (ATCC6538) cultures were propagated to the mid-logarithmic phase respectively. Cells were harvested by centrifugation at $8901 \mathrm{~g}$, washed twice with $10 \mathrm{mM}$, PBS of $\mathrm{pH} 7.4(150 \mathrm{mM}$ $\mathrm{NaCl})$ and resuspended in the same buffer $(1.0 \times$ $\left.10^{6} \mathrm{CFU} / \mathrm{ml}\right)$. E. coli and $S$. aureus cells were incubated with FITC-labelled peptide P52 (FITC-KKKKLKKKLK KLKKKKKKKLLLLL) at $37^{\circ} \mathrm{C}$ for $40 \mathrm{~min}$ (at final concentrations of 160 and $320 \mu \mathrm{M}$ respectively). After incubation, a drop of bacterial suspension was coated on a glass slide. Fluorescence was observed using a confocal laser scanning microscope ${ }^{16}$. Fluorescent images were obtained with a $489 \mathrm{~nm}$ band-pass filter for excitation of FITC.

\section{Transmission electron microscopy of bacterial cells}

Samples containing logarithmic E. coli MG1655 cells in PBS were incubated with peptides of concentration $160 \mu \mathrm{M}$ at $37^{\circ} \mathrm{C}$, corresponding to at least four of their MIC values for $0.5,1$ and $2 \mathrm{~h}$ respectively. Controls containing E. coli MG1655 in PBS were incubated without peptides at $37^{\circ} \mathrm{C}$. Subsequently, all samples were centrifuged at $7300 \mathrm{~g}$ for $1 \mathrm{~min}$. The pellets were fixed using $2.5 \%$ glutaraldehyde and stored at $4{ }^{\circ} \mathrm{C}$ overnight. A drop containing the bacteria was deposited onto a carboncoated grid and negatively stained with $2 \%(\mathrm{w} / \mathrm{v})$ purified terephthalic acid (PTA) ( $\mathrm{pH} 7.0)$. The grids were examined using an electron microscope (JEM 1400) ${ }^{17}$.

\section{Scanning electron microscopy of cancer cells}

The A549 cells were plated in 24-well, flat-bottom, tissue-culture plates containing sterile circular coverslips and cultured overnight. Then, the cells were treated with indicated peptides and concentrations up to $2 \mathrm{~h}$. They were then washed with PBS twice and fixed with $2.5 \%$ glutaraldehyde $(\mathrm{v} / \mathrm{v})$ for $1 \mathrm{~h}$ at $4^{\circ} \mathrm{C}$. Then cells were washed again with PBS followed by dehydration with incremental increases of ethanol $(30 \%, 50 \%, 70 \%, 80 \%$, $90 \%$ and $100 \%)$. After critical-point drying and goldcoating, the samples were observed using SEM ${ }^{18}$.

All figures were prepared using ACDSee 7.0 and Adobe Photoshop 8.0.1.

\section{Results and discussion}

\section{Antibacterial and anticancer activities and leucine insulator}

Over 100 peptides were synthesized by a commercial vender without a priori consideration of any physicochemical properties and analysed for their antibacterial and anticancer efficacy. The (leucine/lycine) $n,(\mathrm{~L} / \mathrm{K}) n$ 


\section{RESEARCH ARTICLES}

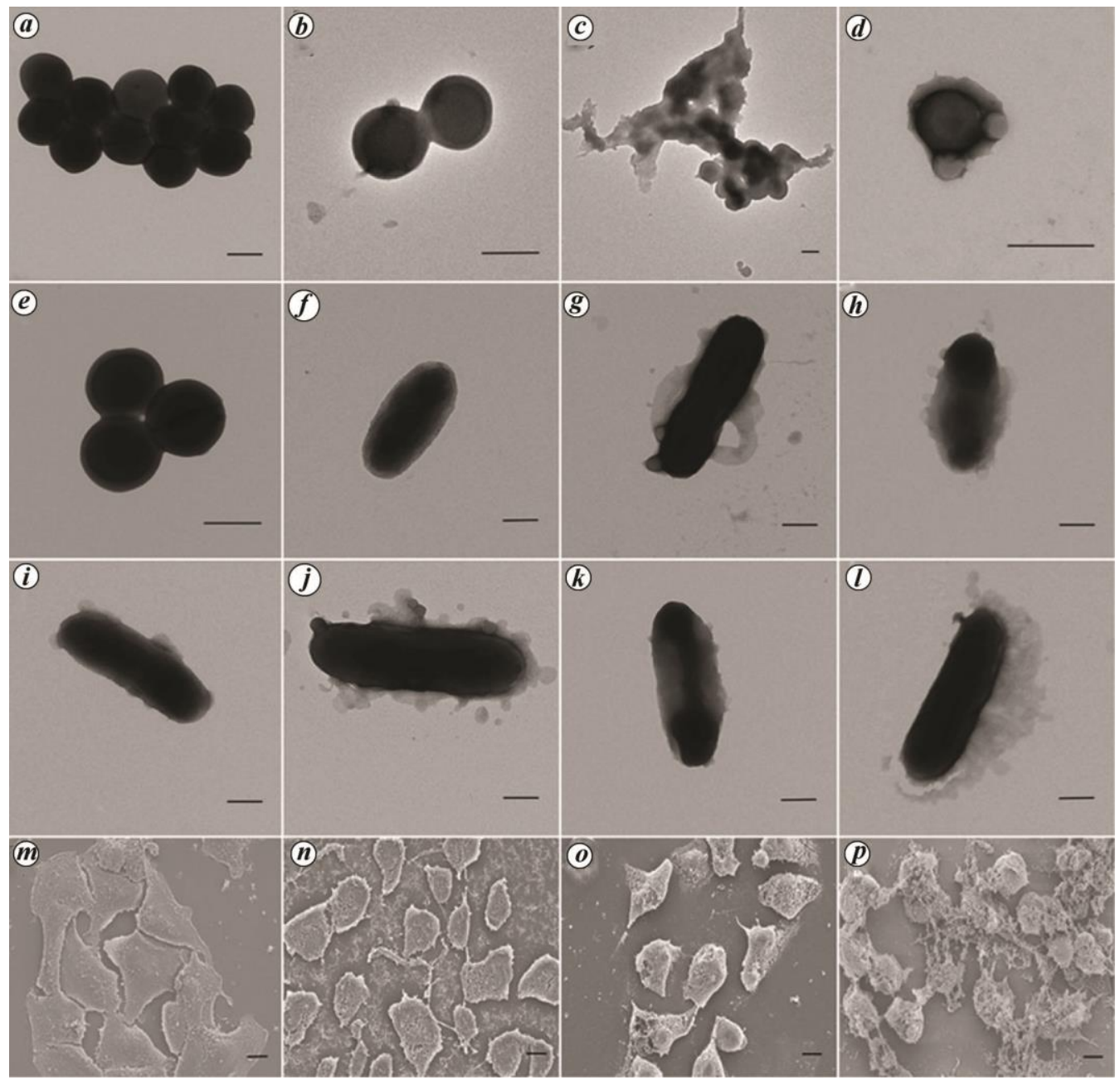

Figure 2. Electron microscopy of bacterial and cancer cells after treatment with peptides. Transmission electron microscopy (TEM) of $S$. aureus (ATCC6538) cells upon incubation with leucine predominant antimicrobial peptide P78 at $160 \mu \mathrm{M}$ : (a) control, $(\boldsymbol{b})$ after $30 \mathrm{~min}$ of incubation and $(\boldsymbol{c})$ after $2 \mathrm{~h}$ of incubation. TEM of $S$. aureus (ATCC6538) cells upon incubation with $160 \mu \mathrm{M}$ antimicrobial peptide P145: (d) after 30 min of incubation and $(\boldsymbol{e})$ after $2 \mathrm{~h}$ of incubation. TEM of Escherichia coli MG1655 cells upon incubation with $160 \mu \mathrm{M}$ AMPs: $(\boldsymbol{f})$ control; $(g)$ after 30 min of incubation with P54; $(\boldsymbol{h})$ after $2 \mathrm{~h}$ incubation with P54; $(\boldsymbol{i})$ after $30 \mathrm{~min}$ of incubation with P83; $(\boldsymbol{j})$ after $2 \mathrm{~h}$ of incubation with P83; $(\boldsymbol{k})$ after $30 \mathrm{~min}$ of incubation with P145 and $(\boldsymbol{l})$ after $2 \mathrm{~h}$ of incubation with P145. Scanning electron microscopy of lung cancer cells A549 upon incubation with $100 \mu \mathrm{M}$ peptides: $(\boldsymbol{m})$ control, $(\boldsymbol{n})$ peptide $\mathrm{P} 45$ at $0.5 \mathrm{~h},(\boldsymbol{o})$ peptide $\mathrm{P} 45$ at $2 \mathrm{~h}$ and (p) P129 at $2 \mathrm{~h}$. Scale bar: $500 \mathrm{~nm}$ for bacteria and 10,000 $\mathrm{nm}$ for cancer cells.

and (phenylalanine/lysine) $n,(\mathrm{~F} / \mathrm{K}) n$ peptides were potent against bacteria (Supplementary Figure 1). The $(\mathrm{F} / \mathrm{K}) n$ peptides were more effective than the $(\mathrm{L} / \mathrm{K}) n,(\mathrm{I} / \mathrm{K}) n$ and $(\mathrm{V} / \mathrm{K}) n$ peptides against $E$. coli in LB $(P=0.000$, Kruskal-Wallis test, two-tailed); $(\mathrm{L} / \mathrm{K}) n$ peptides were also more efficacious than (isoleucine/lysine) $n,(\mathrm{I} / \mathrm{K}) n$ and (valine/lysine) $n, \quad(\mathrm{~V} / \mathrm{K}) n$ peptides against $E$. coli $(P=0.000$, Kruskal-Wallis test, two-tailed); $(\mathrm{L} / \mathrm{K}) n$ and $(\mathrm{F} / \mathrm{K}) n$ peptides showed no difference against $P$. aerugenosa (0.833, Kruskal-Wallis test, two-tailed), but both were more effective than $(\mathrm{I} / \mathrm{K}) n$ and $(\mathrm{V} / \mathrm{K}) n$ peptides $(P=0.000$, Kruskal-Wallis test, two-tailed).

The differences in antibacterial effects of $(\mathrm{L} / \mathrm{K}) n$ peptides in the presence and absence of $1 \% \mathrm{NaCl}$ were statistically significant $(P=0.000$, Greenhouse-Geisser correction, general linear model repeated measures). The $(\mathrm{L} / \mathrm{K}) n$ peptides were generally slightly stronger in the presence than absence of $\mathrm{NaCl}$. The differences of $(\mathrm{F} / \mathrm{K}) n$ peptides in the presence and absence of $1 \% \mathrm{NaCl}$ were modest but statistically significant $(P=0.000$, GreenhouseGeisser correction, general linear model repeated measures). The antibacterial efficacy of the $(\mathrm{V} / \mathrm{K}) n$ and (I/K) $n$ peptides was stronger at $40 \mu \mathrm{M}$ when the assays were conducted in $\mathrm{NaCl}$-containing $\mathrm{LB}$ medium than in the presence of $\mathrm{NaCl}$ versus in the absence of $\mathrm{NaCl}(P=$ $0.000, P=0.000)$ respectively, $n=3$, general linear model repeated measures). However, $(\mathrm{V} / \mathrm{K}) n$ peptides, and to a lesser extent $(\mathrm{I} / \mathrm{K}) n$ peptides, performed poorly in $1 \%$ $\mathrm{NaCl}$-containing LB medium than in $\mathrm{NaCl}$-free $\mathrm{LB}$ medium at higher peptide concentrations (data not shown). 


\begin{tabular}{|c|c|c|c|}
\hline & Anticancer peptide & Insulator & Antibody CDR \\
\hline P45 & LLLLLLKKKLKKKLKKLKKLKKKK & & \\
\hline P129 & LLLLLLKKKLKKKLKKLKKLKKKK & LLLL & ARGGYYTYDDWG \\
\hline P130 & LLLLLLKKKLKKKLKKLKKLKKKK & LLLL & YIYPYNDGTKYNEKFKD \\
\hline P121 & LLLLLLKKKLKKKLKKLKKLKKKK & IIIIIII & ARGGYYTYDDWG \\
\hline P55 & LLLLLKKKKLKKKLKKLKKKKKKK & & \\
\hline P56 & LLLLLKKKKLKKKLKKLKKKKKKKK & LLLL & ARGGYYTYDDWG \\
\hline P57 & LLLLLKKKKLKKKLKKLKKKKKKK & LLLL & YYYPYNDGTKYNEKFKD \\
\hline P58 & & & ARGGYYTDDWG \\
\hline P59 & & & YIYPYNDGTKYNEKFKD \\
\hline P75 & LLLLLRRRRRRLRRLRRRLRRRRR & & \\
\hline P111 & LLLLLRRRRRRLRRLRRRLRRRRR & LLLLLLLLLLLLLLLL & ARGGYYTYDDWG \\
\hline P72 & RRRRLRRLRLRRLRRLLRLLRRRR & & \\
\hline P128 & RRRRLRRLRLRRLRRLLRLLRRRR & LLLLLLLLLLLLLLLL & ARGGYYTYDDWG \\
\hline P54 & KKKKLKKLKLKKLKKLLKLLKKKK & & \\
\hline P63 & LLLLLRRLRLRRLLRRLLLRRLRR & & \\
\hline P66 & LLLLLLLLRRRRRRRRLRRLRRRR & & \\
\hline P68 & LLLRRRRRRLRRLRRRLRRRRRRR & & \\
\hline P71 & RRRRLRRRLRRLRRRRRRRLLLLL & & \\
\hline P118 & АAАAАAKKKLKKKLKKLKKLKKKK & AAAAA & ARGGYYTYDWG \\
\hline
\end{tabular}

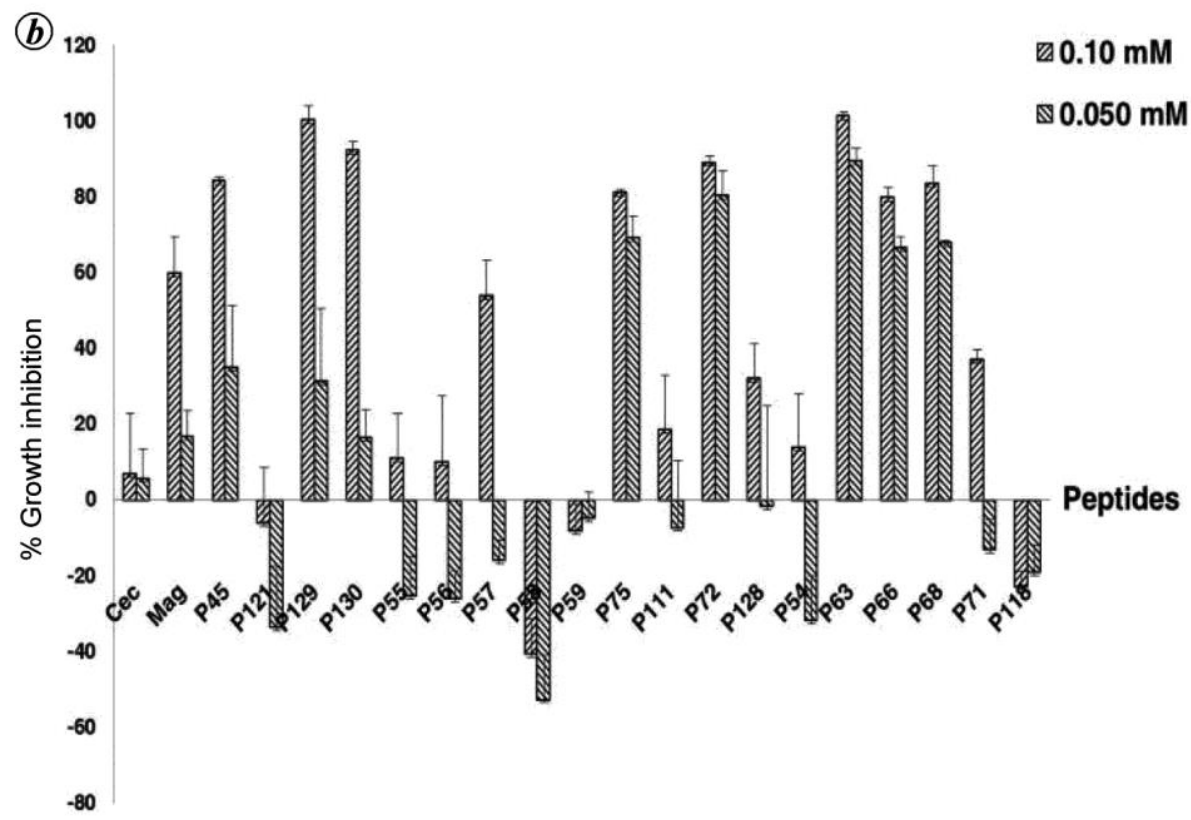

Figure 3. Inhibition rates against lung carcinoma A549 cells using MTT assays. $\boldsymbol{a}$, Tumour targeting peptides consist of an antimicrobial peptide and a CDR fragment of a monoclonal antibody against CD47 receptor separated by a consecutive leucine insulator. P129 carries CDR3, whereas P130 carries CDR1. $\boldsymbol{b}$, Inhibition rates against lung carcinoma A549 cells. Experiments were performed in triplicate with one standard deviation shown. Statistical analysis on treatments at $100 \mu \mathrm{M}$ was performed $(n=3$, growth rate), as follows: P129 $(P=0.023$ versus control P58); P63 ( $P=0.018$ versus P58; $P=0.047$ versus P118; two-tailed, Kruskal-Wallis test).

Given the permutations of both $\mathrm{L}$ and $\mathrm{D}$ isomers in the consensus sequences $(\mathrm{L} / \mathrm{F} / \mathrm{K}) n$, these AMPs with various lengths have enormous combinatorial diversity. Electron microscopic analysis showed that the leucine-predominant P78 peptide disintegrated the $S$. aureus cells after $2 \mathrm{~h}$ of incubation (Figure $2 c$ ), while the $E$. coli cells remained largely intact after 30 min treatment with the lysine-predominant peptides P54, P83 and P145 (Figure $2 f-l)$. P145 also did not disrupt the $S$. aureus cells after treatment (Figure $2 d-e$ ), suggesting that an alternative cell damage mechanism may be involved. Conservative replacements of all leucine residues with valine or isoleucine yielded less active peptides (Supplementary Tables 1 and 2, Figure 1). P125 and P127 harbouring only leucine insulator and antibody CDR were ineffective against the bacteria (Supplementary Figure 1).

Tumour targeting peptides, consisting of an antimicrobial peptide described above and a CDR fragment of 
an antibody against CD47 receptor ${ }^{19,20}$ separated by a four consecutive leucine insulators, retained activities against bacteria, as shown with peptides P56 and P57 versus their predecessor P55, or with peptides P131 and P132 versus their predecessor P54 (Supplementary Figure 1 and Table 2). In the initial tests for anticancer potential, some of the peptides were more potent than the prototype antibacterial peptides against lung carcinoma A549 cells at modest concentrations (Figure 3), indicating that the designed nanometre-biomissiles (nano-biomissiles) represent an effective strategy targeting at the cell surface markers. CD47 receptors are more abundant in cancer cells than in normal cells, although they are broadly distributed in the body, including erythrocytes ${ }^{19}$. One tumour targeting peptide P129 recorded complete (100\%) eradication of cancer cells at $100 \mu \mathrm{M}$ as determined by MTT assays, versus $84.2 \%$ by its predecessor peptide $\mathrm{P} 45$ (Figure $3 b$ ). The control peptides P58 and P59 recorded growth enhancement activities toward cancer cells, which were antibody $\mathrm{CDR}$ alone $(P=0.023, \mathrm{P} 129$ versus control P58 at $100 \mu \mathrm{M}$, Kruskal-Wallis test, two-tailed). The anti-E. coli activities of tumour targeting peptides P129, $\mathrm{P} 130, \mathrm{P} 56$ and P57 derived from $(\mathrm{L} / \mathrm{K}) n$ peptides at $40 \mu \mathrm{M}$ correlated well with anticancer activities at $100 \mu \mathrm{M}$ in MTT assays $(P=0.753, n=3$, covariance analysis). Electron microscopic analysis indicated that P129 resulted in more significant damage on A549 lung cancer cells than P45 (Figure $2 n-p$ ). Six consecutive isoleucines in P121 or phenylalanines in P120 were much less effective as insulators in nano-biomissiles when they were tested for antibacterial or anticancer activities (Figure 3; Supplementary Figure 1). (L/F/K)n peptides were highly active in antibacterial assays, including observations on a multidrug-resistant $S$. aureus strain Y5 (Supplementary Figure 2).

\section{HT29 subcutaneous transplantation tumour animal models}

Low doses of the CD47 targeting peptide P129 exhibited potent anticancer activities in mouse experiments. The HT29 tumour animal model was subjected to tumour growth inhibition test (Figure 4). Mice of the PBS mock control showed poor appetite and adverse physiological responses, while the physiological conditions of the P129 treatment group were significantly better. P129 substantially inhibited tumour growth $(P<0.05$, general linear model repeated measures; Figure $4 a$ ). Mice were sacrificed after the treatment regimen and the tumour sections were subjected to $\mathrm{H} \& \mathrm{E}$ staining (Figure $4 c$ and $d$ ) and TUNEL assay (Figure $4 e-f$ ). The tumour cells of the mock control showed high density with bigger and atypical nuclei, indicating that these cells were in proliferation. The tumour cells of the P129 treatment group exhibited low density with nuclei pyknosis, suggesting that the peptide could inhibit tumour cell growth and promote cell apoptosis. The TUNEL staining of PBS mock control was virtually DAB-negative, and cells of the P129 treatment group were stained extensively brown, suggesting that tumours of the P129 treatment group experienced wide cell apoptosis. The mock control group recorded one dead mouse on day 23, whereas all mice in the peptide treatment group survived and responded well to the regimen.

\section{Membrane permeability and hemolysis assays}

Designed with around $50-70 \%$ basic amino acids and the rest hydrophobic amino acids, these peptides usually caused no substantial changes of membrane permeability (Supplementary Figure $3 a-c$ ), and some peptides were weakly hemolytic at a concentration of $10 \mu \mathrm{M}$ (Supplementary Figure 4). Attenuation of hemolysis by terminal replacements with alanine or valine on the peptides derived from P45 or P149 was observed, whereas minor or modest changes of anti-E. coli activities in these peptides were recorded (Supplementary Figure 5). Marked attenuation of hemolysis by P45-3 with terminal alanine substitution on P129 suggests that biosafety profile of a peptide can be improved (Supplementary Figure $4 c$ ). Confocal laser-scanning microscopic examination indicated that the FITC-labelled peptide P52 was able to readily penetrate the membranes and accumulate in the cytoplasm of both E. coli and $S$. aureus cells (Supplementary Figure $3 d$ and $e$ ).

The highly enriched particular amino acids in the peptides under study suggest that they are influenced by the chemical properties of the over-represented amino acid residues. The membrane permeability assay of the peptides indicates that they are distinct from the conventional membrane-lytic antimicrobial peptides. Previous studies corroborated the interactions between carbonyl group and $\gamma$-methyl groups in valine ${ }^{3,4}$. Electron delocalization on $\gamma$-methyl groups of isoleucine and valine has been suggested by NMR chemical shifts ${ }^{21,22}$, coupling constants $^{3,4,23,24}$, and red shifts of the UV and CD spectra relative to those of leucine ${ }^{25,26}$. The hydrogen bond or other types of secondary weak bonds with carbonyl oxygen could be enhanced by van der Waals forces between the positively charged carbonyl carbon and $\sigma-\sigma$ hyperconjugated $\beta$-carbon-H. As previously reported, the van der Waals interactions between side chains and the local backbone of proteins determine the intrinsic propensities for $\beta$-sheet formation, notably for $\beta$-branched amino acids ${ }^{5}$. This may be achieved through enhanced hydrogen bonds.

Other factors that support the above are the large dipole moment ${ }^{27}$, and longer and shorter bond lengths respectively, in $\mathrm{C}=\mathrm{O}$ and $\mathrm{C} \beta-\mathrm{C} \gamma$ in valine than leucine, or in $\mathrm{C}=\mathrm{O}$ and $\mathrm{C} \beta-\mathrm{C} \gamma 2$ in isoleucine than leucine ${ }^{7,8}$. Strong 

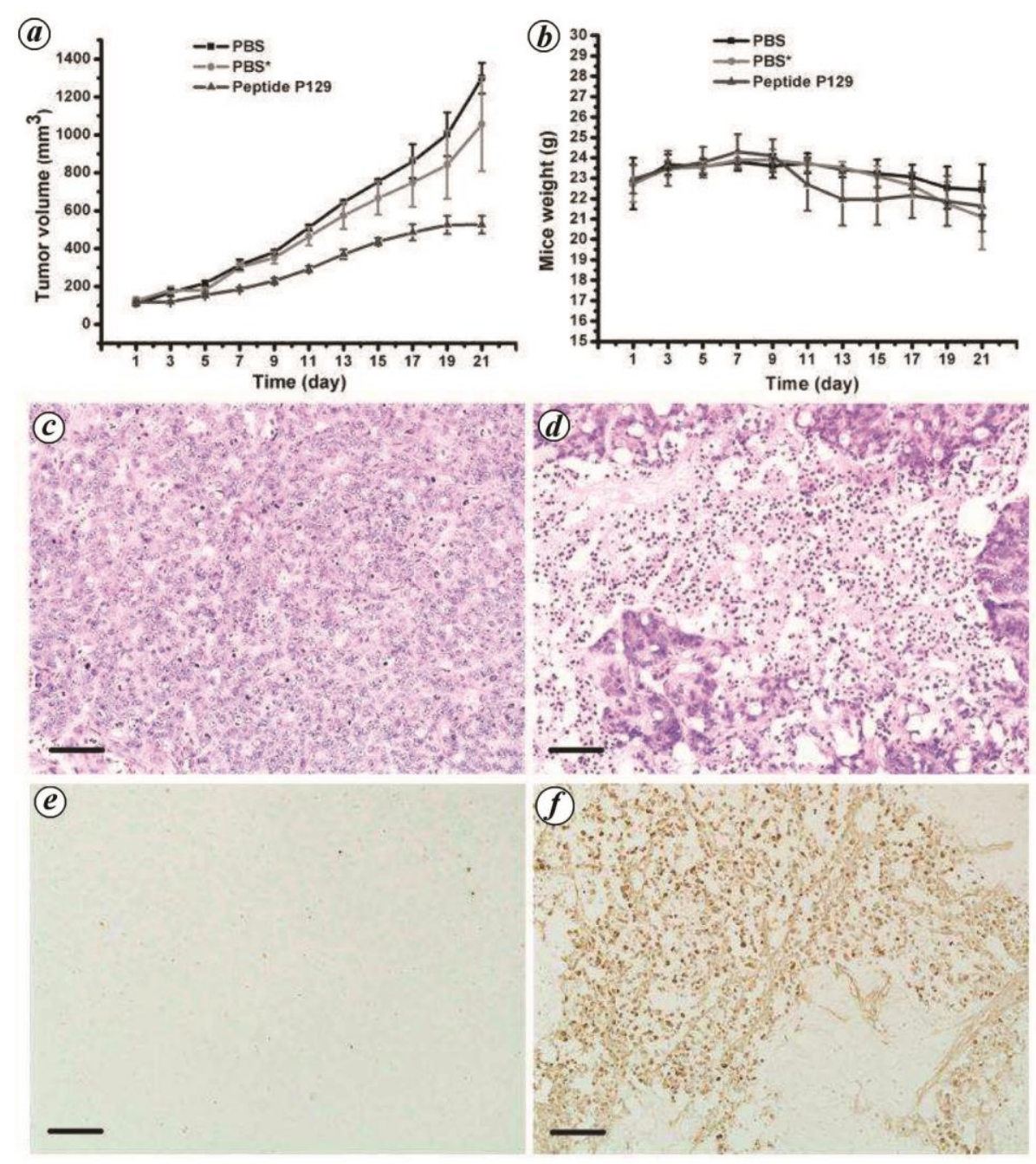

Figure 4. Treatment of tumours with peptide P129 in a HT29 subcutaneous transplantation tumour mouse model. $\boldsymbol{a}$, Tumour growth curves after treatment with PBS (mock control) and P129 with $1.2 \mathrm{mg}$ peptide per injection per two days starting at day 1. Means and standard errors are shown. One mouse bearing a small tumour in the control group died of cancer at day 23. After peptide treatment $(n=5$ in the peptide treatment group), tumour volumes were reduced significantly $(P=0.024$ when the dead mouse was included (PBS*, $n=4$ in the mock control group); $P=0.000$ when the dead mouse was removed from the mock control data (PBS, $n=3)$ ). $\boldsymbol{b}$, Body weight curves after treatment with vehicle PBS and peptide P129. After peptide treatment, body weight changes were not significant $(P=0.832$ (PBS*), $P=0.719$ (PBS) versus peptide treatment group). $(\boldsymbol{c}, \boldsymbol{d})$ Histological characteristics and $(\boldsymbol{e}, \boldsymbol{f})$ TUNEL assays of HT29 tumour tissues $(\boldsymbol{d}, \boldsymbol{f})$ after or $(\boldsymbol{c}, \boldsymbol{e})$ without treatment with peptide P129. In H\&E staining, blue indicates nuclei and red indicates extracellular matrix and cytoplasm. In TUNEL analysis, brown and green stains indicate apoptotic and normal cells respectively. Scale bar: $50 \mu \mathrm{m}$.

hydrogen bonding and secondary weak chemical bonding to the carbonyl oxygen of the carboxyl groups were reported to be related to longer than normal $\mathrm{C}=\mathrm{O}$ bond lengths ${ }^{8,28}$. There was a marked red shift of the CD spectrum around $200 \mathrm{~nm}$ of isoleucine versus leucine at $\mathrm{pH} 2$ relative to $\mathrm{pH} 7$ (ref. 25 ), which is due to the $n-\pi^{*}$ transitions of the carboxyl group ${ }^{29}$. The carbonyl carbon of isoleucine was the most shielded among the amino acids tested as shown by the chemical shift anisotropy of $\sigma_{22}$ element of the tensor ${ }^{8}$, which supports the presence of stronger hydrogen bonds with the carbonyl oxygen in isoleucine than in leucine (Figure 1). When carbonyl oxygen of isoleucines in $(\mathrm{I} / \mathrm{K})$ peptides forms hydrogen bonds with the protons, it does not weaken the anion trapping and bacteriostatic properties of lysine residues. When carbonyl oxygen of isoleucines forms secondary bonds with $\mathrm{K}^{+}$or $\mathrm{Na}^{+}$, it attenuates the peptides when isoleucines are situated within lysine clusters. P61 is a hybrid peptide of $(\mathrm{I} / \mathrm{L} / \mathrm{K})$, and its isoleucine stretch is present only at the peptide terminus; so the effect of isoleucine residues on lysine clusters is less pronounced.

A recent study revealed that leucine replacements at the isoleucine and valine sites have dramatically improved temperature and $\mathrm{pH}$ tolerance of a 
mini-cellulase ${ }^{30}$, perhaps explained by weak interactions between carbonyl oxygen of leucine residues and other molecules in the ambient environment. While non- $\beta$ branched leucines in most proteins are harmless and help form higher-order structures, they can sustain toxic peptides that disrupt or kill cells. Furthermore, $\sigma-\sigma$ hyperconjugation rendered by the $\delta$-methyl groups of leucine may allow inter-molecular van der Waals interactions. This hypothesis is corroborated by NMR chemical shifts ${ }^{21,22}$, bond lengths (7) and leucine zipper motifs facilitating dimerization ${ }^{31}$.

With two $\gamma$-methyl groups valine exhibits longer $\mathrm{C}=\mathrm{O}$ bond length than that of isoleucine, and its carbonyl oxygen may form stronger secondary bonding with divalent cations than the counterpart of isoleucine. Several peptides show potency in the presence of sodium citrate or/and sodium oxalate (Supplementary Figure 1), suggesting the presence of alternative cell death mechanism using organic acids. Oxalic acid was reported to be an important virulence factor produced by phytopathogenic filamentous fungi ${ }^{32}$. Given its role in the formation of kidney stones and other diseases, compounds with similar structures to oxalate such as ethanol, acetic acid, pyruvate and alanine have several of beneficial effects in disease treatment and lifespan extension after moderate intake $^{33-36}$.

As peptides might be degraded over time in vivo, no adverse reactions were observed in the peptide treatment group in the HT29 subcutaneous transplantation tumour mouse model. Drug cocktails can be formulated to take advantage of the unique properties of each peptide. Certain bacteria may be inhibited or killed by ion aggregation, and others might be sensitive to locally formed strong acids. Particular cells might be susceptible to insoluble salts like calcium oxalate. Potent $(\mathrm{L} / \mathrm{K}) n$ peptides were generally active regardless of the culture medium. The design of P44, P45, P46, P47, P48, P49, P50, P51, P52 and P55 was to identify the best terminal anchor of the peptides onto the membranes. The anti-E. coli and anticancer data indicated that peptides with 5-6 leucines at the terminus were effective.

\section{Conclusion}

Tumour targeting peptides consisting of an $(\mathrm{L} / \mathrm{K}) n$ peptide and an antibody CDR fragment separated by a leucine insulator, were effective against cancer cells in in vitro assays and an in vivo mouse model. The immense combinatorial diversity of $(\mathrm{K} / \mathrm{L} / \mathrm{F}) n$ antibacterial peptides warrants further studies.

Conflict of interest: The authors declare that they have no conflict of interest.

1. Hoskin, D. W. and Ramamoorthy, A., Studies on anticancer activities of antimicrobial peptides. Biochim. Biophys. Acta, 2008, 1778(2), 357-375.
2. Giuliani, A., Pirri, G. and Nicoletto, S. F., Antimicrobial peptides: an overview of a promising class of therapeutics. Cent. Eur. J. Biol., 2007, 2(1), 1-33.

3. Suardíaz, R. et al., Theoretical Karplus relationships for vicinal coupling constants around v1 in valine. Chem. Phys. Lett., 2007, 442, 119-123.

4. Chou, J. J., Case, D. A. and Bax, A., Insights into the mobility of methyl-bearing side chains in proteins from (3) $\mathrm{J}(\mathrm{CC})$ and (3)J(CN) couplings. J. Am. Chem. Soc., 2003, 125(29), 89598966.

5. Street, A. G. and Mayo, S. L., Intrinsic beta-sheet propensities result from van der Waals interactions between side chains and the local backbone. Proc. Natl. Acad. Sci. USA, 1999, 6(16), 9074 9076.

6. Wang, Y. et al., Furthering our understandings on coding SNPs: $\beta$-branched amino acids in diseases. Science, 2017; http://science. sciencemag.org/content/350/6265/1262/tab-e-letters.

7. Gould, R. O., Gray, A. M., Taylor, P. and Walkinshaw, M. D., Crystal environments and geometries of leucine, isoleucine, valine and phenylalanine provide estimates of minimum nonbonded contact and preferred van der Waals interaction distances. J. Am. Chem. Soc., 1985, 107(21), 5921-5927.

8. Gu, Z., Zambrano, R. and Mcdermott, A., Hydrogen bonding of carboxyl groups in solid-state amino acids and peptides: comparison of carbon chemical shielding, infrared frequencies, and structures. J. Am. Chem Soc., 2002, 116(14), 6368-6372.

9. He, R. et al., A combinatorial yeast overlay method for the isolation of antibacterial oligopeptides. Proc. Natl. Acad. Sci. India Sect. B, 2014, 84(4), 1069-1075.

10. Steiner, H., Sequence and specificity of two antibacterial proteins involved in insect immunity. Nature, 1981, 292, 246-248.

11. Zasloff, M., Magainins, a class of antimicrobial peptides from Xenopus skin: isolation, characterization of two active forms, and partial cDNA sequence of a precursor. Proc. Natl. Acad. Sci. USA, 1987, 84(15), 5449-5453.

12. Cozens, A. L. et al., Cftr expression and chloride secretion in polarized immortal human bronchial epithelial cells. Am. J. Respir. Cell Mol. Biol., 1994, 10(1), 38-47.

13. Lieber, M. et al., A continuous tumor-cell line from a human lung carcinoma with properties of type II alveolar epithelial cells. Int. J. Cancer, 1967, 17(1), 62-70.

14. Warshamana-Greene, G. S. et al., The insulin-like growth factor-I receptor kinase inhibitor, NVP-ADW742, sensitizes small cell lung cancer cell lines to the effects of chemotherapy. Clin. Cancer Res., 2005, 11(4), 1563-1571.

15. Chen, W. et al., Co-delivery of doxorubicin and siRNA with reduction and $\mathrm{pH}$ dually sensitive nanocarrier for synergistic cancer therapy. Small, 2014, 10(13), 2678-2687.

16. Park, K. H. et al., Cell specificity, anti-inflammatory activity, and plausible bactericidal mechanism of designed Trp-rich model antimicrobial peptides. Biochim. Biophys. Acta, 2009, 1788(5), 1193-1203.

17. Joshi, S. et al., Interaction studies of novel cell selective antimicrobial peptides with model membranes and E. coli ATCC 11775. Biochim. Biophys. Acta, 2010, 1798(10), 1864-1875.

18. Hilchie, A. L. et al., Pleurocidin-family cationic antimicrobial peptides are cytolytic for breast carcinoma cells and prevent growth of tumor xenografts. Breast Cancer Res., 2011, 13(5), R102.

19. Willingham, S. B. et al., The CD47-signal regulatory protein alpha (SIRPa) interaction is a therapeutic target for human solid tumors. Proc. Natl. Acad. Sci. USA, 2012, 109(17), 6662-6667.

20. Yasufumi, K. et al., Humanized anti-CD47 antibody. 2006, Patent No. EP1693385.

21. Zhang, H., Neal, S. and Wishart, D. S., RefDB: a database of uniformly referenced protein chemical shifts. J. Biomol. NMR, 2003, 25, 173-195. 
22. Wishart, D. S. et al., ${ }^{1} \mathrm{H},{ }^{13} \mathrm{C}$ and ${ }^{15} \mathrm{~N}$ random coil NMR chemical shifts of the common amino acids. I. Investigations of nearestneighbor effects. J. Biomol. NMR, 1995, 5(1), 67-81.

23. Penkett, C. J. et al., NMR analysis of main-chain conformational preferences in an unfolded fibronectin-binding protein. J. Mol. Biol., 1997, 274(2), 152-159.

24. Smith, L. J. et al., Analysis of main chain torsion angles in proteins: prediction of NMR coupling constants for native and random coil conformations. J. Mol. Biol., 1996, 255(3), 494-506.

25. Nishino, H. et al., The $\mathrm{pH}$ dependence of the anisotropy factors of essential amino acids. J. Chem. Soc. Perkin Trans. 2, 2002, 23(3), $582-590$.

26. Matsuo, K. et al., Vacuum-ultraviolet circular dichroism of amino acids as revealed by synchrotron radiation spectrophotometer. Chem. Lett., 2002, 5(8), 826-827.

27. Ganesan, A., Brunger, M. J. and Wang, F., A study of aliphatic amino acids using simulated vibrational circular dichroism and Raman optical activity spectra. Eur. Phys. J. D, 2013, 67(11) 229.

28. Ichikawa, M., The effect of hydrogen bonding on the bond lengths and angles in the carboxyl group. J. Chem. Crystallogr., 1979, 9(2), 87-105.

29. Snyder, P. A., Vipond, P. M. and Johnson, W. C., Circular dichroism of the alkyl amino acids in the vacuum ultraviolet. Biopolymers, 1973, 12(5), 975-992.

30. Xiao, Y. et al., Neurospora crassa tox-1 gene encodes a pH- and temperature-tolerant mini-cellulase. J. Agric. Food Chem., 2016, 64(23), 4751-4757.

31. E, Z. G., Zhang, Y. P., Zhou, J. H. and Wang, L., Mini review roles of the bZIP gene family in rice. Genet. Mol. Res., 2014, 13(2), 3025-3036.

32. Godoy, G., Steadman, J. R., Dickman, M. B. and Dam, R., Use of mutants to demonstrate the role of oxalic acid in pathogenicity of
Sclerotinia sclerotiorum on Phaseolus vulgaris. Physiol. Mol. Plant Pathol., 1990, 37(3), 179-191.

33. Chow, F. C., Dysart, M. I., Hamar, D. W. and Udall, R. H., Control of oxalate urolithiasis by DL-alanine. Invest. Urol., 1975, 13(2), 113-116.

34. Chuang, M. H. et al., The lifespan-promoting effect of acetic acid and Reishi polysaccharide. Bioorg. Med. Chem., 2009, 17(22), 7831-7840.

35. Paganini-Hill, A., Kawas, C. H. and Corrada, M. M., Type of alcohol consumed, changes in intake over time and mortality: the Leisure World Cohort Study. Age Ageing, 2007, 36(2), 203-209.

36. An, S. et al., Functional duality of ethanol on cancer. Med. Hypotheses, 2019, 122, 124-125.

ACKNOWLEDGEMENTS. This work was supported by grants from the Science and Technology Transformation Programme of Sun Yat-sen University of China (No. 33000-18843234) and Guangzhou Science and Technology Programme (No. 201804010328) to Q. L.; China Agriculture Research System (47), The National Key Technology R\&D Programme (2012BAD17B03), and The National Key Technology R\&D Programme (2011BAD13B10) to J. H. and The National Natural Science Foundation of China (21601209) to Y. W. We thank D. Augustyniak for the ML-35 strain and Guangchuang Yu for help with statistical analysis. We also thank Bo Yu, Li Xu, Jing Li, Fan Yang and Xiang Zhu for suggestions and technical assistance and Ms. Yan Shi at Sun Yat-sen University for editorial assistance. Qiuyun Liu, Jianguo $\mathrm{He}$ and Wenliang Zhou co-supervised the work. Meng Xing, Xiaoxia Li and Shanshan An contributed equally to this project.

Received 21 March 2019; revised accepted 7 October 2020

doi: $10.18520 / \mathrm{cs} / \mathrm{v} 120 / \mathrm{i} 1 / 177-185$ 